Original articles

\title{
Prenatal diagnosis of osteogenesis imperfecta by identification of the concordant collagen 1 allele
}

Jennifer R Lynch, Donald Ogilvie, Linda Priestley, Carolyn Baigrie, Roger Smith, Peter Farndon, Bryan Sykes

\begin{abstract}
Dominantly inherited osteogenesis imperfecta is consistently linked to the two loci encoding the $\alpha 1$ and $\alpha 2$ subunits of collagen 1 , the predominant bone collagen. We have performed several prenatal diagnoses based on identification of the segregating allele at the concordant locus in chorionic villus samples both in families where the linkage can be independently shown and in those where it cannot. Especially in the latter category, calculation of the final risk must incorporate an estimate of genetic heterogeneity within the OI population to give a prior probability of linkage. This figure can then be modified for each family by additional information from concordant meioses.
\end{abstract}

The osteogenesis imperfecta (OI) phenotype varies, more or less continuously, from cases which die soon after birth at one extreme to near normality at the other. The phenotype results from mutations in either of the two genes, COL1A1 and COL1A2, encoding collagen 1 , which is an important structural protein in bone. The severity seems to depend on which gene is involved and on the nature of the mutation. ${ }^{1}$ All

University of Oxford, Institute of Molecular Medicine, John Radcliffe Hospital, Headington, Oxford OX3 9DU. D Ogilvie, L Priestley, C Baigrie, B Sykes

Nuffield Department of Clinical Medicine, John Radcliffe Hospital, Oxford OX3 9DU.

J R Lynch, R Smith

Clinical Genetics Unit, Birmingham Maternity Hospital, Edgbaston, Birmingham B15 2TG.

$P$ Farndon

Correspondence to Dr Sykes.

Received for publication 27 July 1990

Revised version accepted for publication 23 August 1990. unrelated cases result from separate mutational events and almost always involve only one allele. Thus, most cases are heterozygotes and the disease is inherited as an autosomal dominant trait. The disease is sufficiently severe for there to be a demand for prenatal diagnosis from parents at risk of having an affected child.

Sillence $e t a l^{2}$ divided OI into four categories (types I to IV) based largely on phenotype. Type II is lethal in the perinatal period. ${ }^{3}$ Type III is very severely deforming and, although patients can survive into their teens and beyond, they only rarely have children. ${ }^{4}$ Types I and IV are less severe and almost all pedigrees with affected members in more than one generation are drawn from these two Sillence groups.

As expected for a disadvantageous dominant trait, the birth frequency in the population, of about 1 per 10000 for all OI, is maintained by a high rate of new mutation. This is probably the result of the vulnerability of the collagen gene/protein system to mutation. ${ }^{5}$ The disease can then be inherited as an autosomal dominant trait with a $50 \%$ risk to the offspring of an affected parent. Not surprisingly, the disease in most families is the result of a fresh mutation that has arisen in the recent past.

Three techniques are currently available for prenatal diagnosis. High resolution ultrasound scans at 16 to 18 weeks should pick up all type II fetuses and some type III fetuses. ${ }^{6}$ Type IV and I fetuses are only very rarely detected by ultrasound. Some progress has been made in diagnosing OI from analysis of the collagen synthesised by cultured chorionic villus fibroblasts. ${ }^{7}$ When the protein abnormality is obvious, the technique can work well. However, more often than not, the protein abnormality is less clear cut, especially among the less severely affected fetuses that are likely to escape detection by ultrasound.

The third method, which this paper addresses, works by identification of the mutant collagen 1 allele in the affected parent and then in the fetus.

Collagen 1 is composed of two different subunits 
encoded at the non-syntenic loci COL1A1 (17q21.3122.15, $\alpha 1$ subunit) and COL1A2 (7q21.3-22.1, $\alpha 2$ subunit). Each collagen 1 molecule consists of two $\alpha 1$ and one $\alpha 2$ subunits.

Segregation analysis in large dominant pedigrees of the two loci has shown that the disease can be linked to either gene. ${ }^{8-10}$ Prenatal diagnosis based on identification of the concordant allele is straightforward when the pedigree is sufficiently large for a high positive lod score to be generated independently in that family. However, in smaller families, which make up the bulk of cases requesting prenatal diagnosis, this is not possible and the probability that the family is unlinked to either locus has to be brought into the risk calculation. To provide an estimate of the probability that a previously unstudied family is linked, a collaborative study analysed the segregation of both loci in 38 large families. ${ }^{11}$ No families were found that were discordant at both COLIA1 and COL1A2, therefore providing no direct evidence of any unlinked families within the study set. Additionally, the data allowed $95 \%$ confidence limits of the proportion of linked families in the OI population to be set between 0.91 and 1.00 .

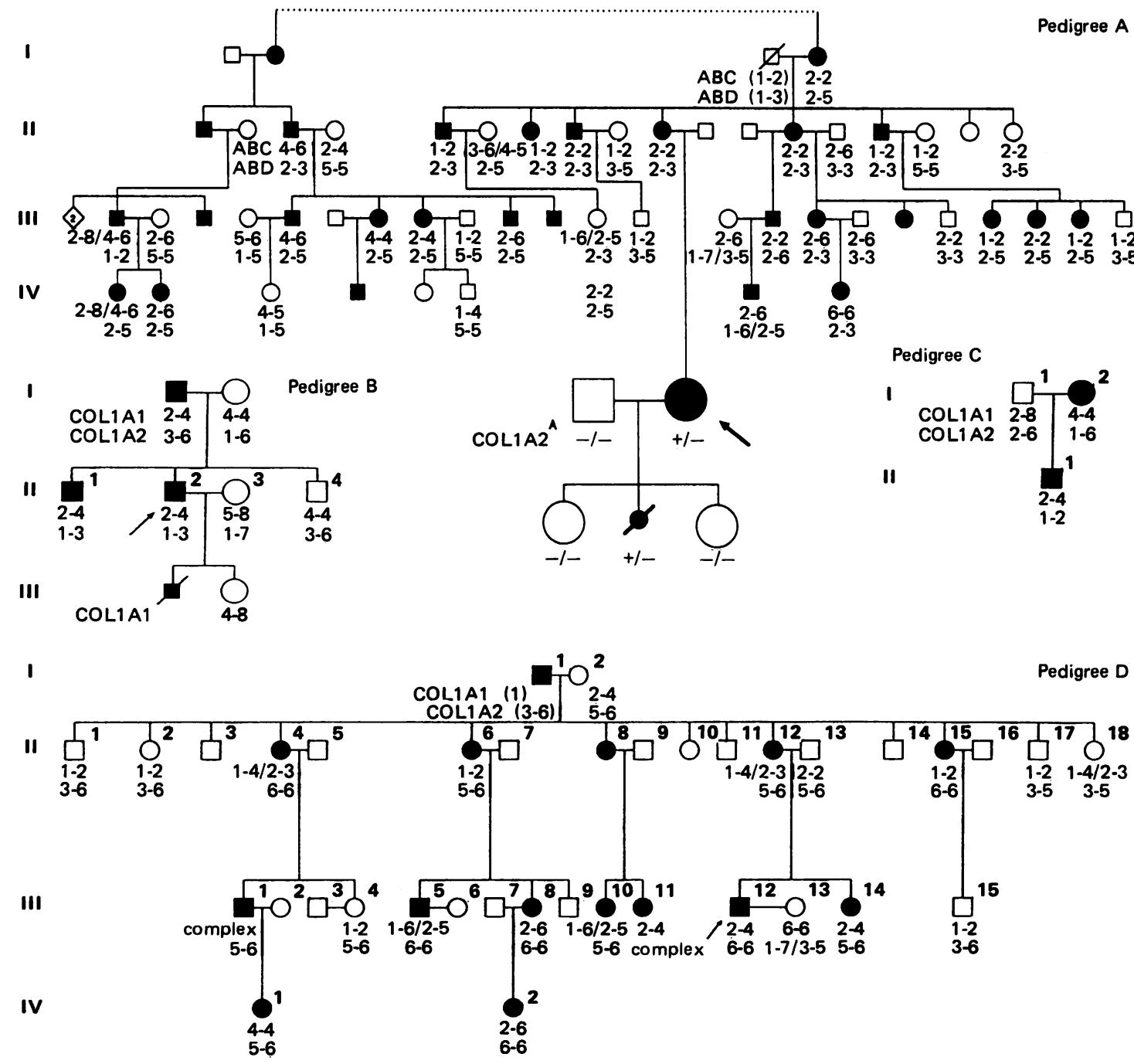

Figure 1 Pedigrees and their COLIAI (upper) and COL1A2 (lower) genotypes. Where alleles are denoted by numbers they comply with the system shown in table 1 . Alternative genotypes which could not be distinguished by referring to relatives are separated by a slash. Where letters are used they refer to enzymelprobe systems in fig 2. Genotype 'complex' is given for subjects who are heterozygous for all markers at the locus and whose potential genotypes (1-8/2-7/3-6/4-5) could not be distinguished. Genotypes in brackets are deduced. 
We have operated a prenatal diagnostic service for dominantly inherited OI since December 1987. This paper illustrates the methods and their limitations, by using four pedigrees.

\section{Patients and methods}

Pedigree A (fig 1) is a large Icelandic family which formed part of the large study referred to above. ${ }^{11}$ The proband was typical of other affected family members in being of normal height, with normal sclerae and no deformity, but large numbers of fractures, in her case over 50 . The phenotype was classified as OI type IV according to the Sillence system. The proband and her husband requested prenatal diagnosis as a result of having been included in the original linkage study.

Pedigree B (fig l) is a small Sillence type IA family from the UK. The proband, II.2, is not severely affected, having had only 'several' long bone fractures up to the age of 12 and only two or three since then. He has blue sclerae, normal teeth, and progressive mixed deafness which began in his thirties. However, his older brother, II.1, has had at least 60 fractures and, partly because of the severity of the disease in his brother, the proband and his wife decided against having a family. Nevertheless, II. 3 became pregnant and, at 22 weeks, after she reported feeling that her baby had broken a bone, a scan showed what appeared to be a fracture of the right femur with the fetus having immobile lower limbs. The pregnancy was terminated. Histological examination of the bones showed columnisation of the enlarging chondrocytes to be irregular and poorly organised. The fracture was confirmed. The decision to have no further children was reinforced. II. 3 became pregnant once more and was on the point of terminating this pregnancy whatever the outcome when the possibility of prenatal diagnosis became available. CVS and blood samples from relatives were analysed simultaneously.

Pedigree C (fig 1) is a Sillence type IA nuclear family from the UK with a mother and son with normal stature, blue sclerae, and few fractures. The parents decided against having further children unless they could be certain of having an unaffected child.

Pedigree D (fig 1) is a large UK family. The proband was 25 years old and his phenotype was typical type IV with short stature and white sclerae. He was born with fractures and had suffered more than 50 since. He had moderately severely deformed limbs and was dependent on a wheelchair though not confined to it. He requested an investigation with a view to prenatal diagnosis. $\mathrm{He}$ and his wife were not prepared to have children unless prenatal diagnosis was available to them.

Three dimorphic restriction site marker systems were used at both $C O L 1 A 1$ and $C O L 1 A 2$ (fig 2) to distinguish alleles. Information from separate sites was combined as haplotypes according to the numerical notation shown in table 1 .

The first stage of the diagnosis was to genotype all the family members in order to identify the mutant locus and allele. Next, informative dimorphisms were tested in the chorionic villus sample.
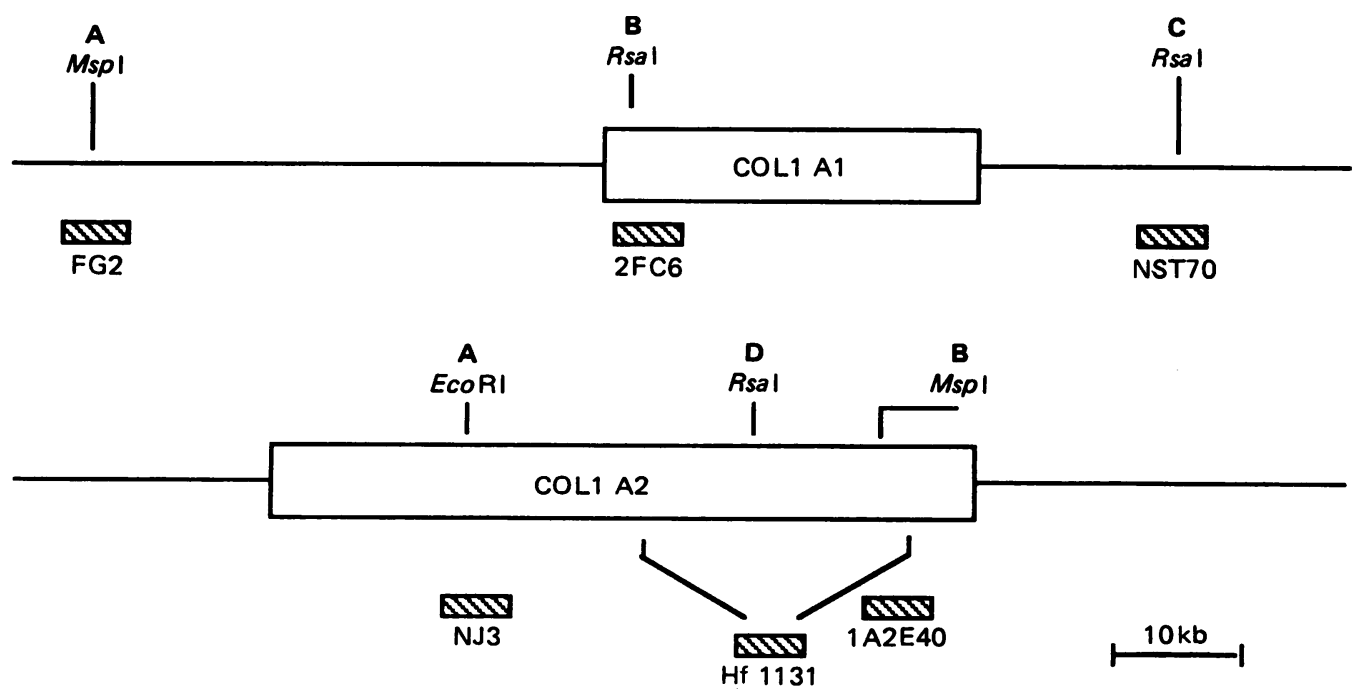

Figure 2 Enzyme/probe systems at COLIAI and COL1A2. Additional information on each site including allele frequencies and fragment sizes is published elsewhere. ${ }^{12}$ I3 
Table 1 Haplotype notation. Combinations of separate variants at both COLIA1 and COLIA2 used to construct the numerical haplotypes in fig 1 . Sites at each locus refer to the restriction sites in fig 2.

\begin{tabular}{rrrrr}
\hline & & \multicolumn{3}{c}{ Site } \\
\hline COLIAI & A & B & C \\
COLIA2 & A & B & D \\
Haplotype & 1 & + & + & + \\
& 2 & + & + & - \\
& 3 & + & - & + \\
& 4 & + & + & - \\
& 5 & - & + & + \\
& 6 & - & - & + \\
& 7 & - & - & - \\
\hline 8 & - & &
\end{tabular}

\section{Results}

PEDIGREE A

The family study showed that $C O L 1 A l$ was discordant but allele 2 at $C O L 1 A 2$ cosegregated with the disease with a lod score of $7 \cdot 7$ at zero recombination distance. With such a high positive lod score there was no need to refer to any other data in order to assign $C O L 1 A 2$ allele 2 as the mutant. The genotype of the proband $(\mathrm{A}+-\mathrm{B}++\mathrm{C}+-)$ and her husband $(\mathrm{A}++\mathrm{C}--)$ meant that dimorphic system $A(E c o R I \times N J 3)$ was fully informative. Only this system was used to genotype the fetal DNA. We have performed three prenatal diagnoses for this couple and the results are shown in fig 3. Our predictions for pregnancies 1 and 3 were that the fetus was not affected and they resulted in the birth of normal girls. Our prediction for pregnancy 2 was that the fetus had inherited the OI gene and the pregnancy was terminated. The probability of linkage to allele 2 calculated from the lod score in the proband was given as 0.9999 . The

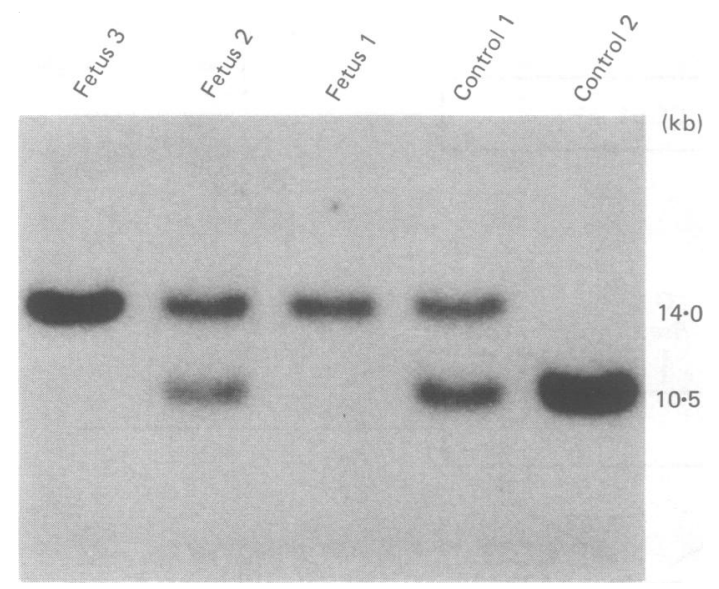

Figure 3 Bands at $14 \cdot 0 \mathrm{~kb}$ and $10 \cdot 5 \mathrm{~kb}$ are products of the $(-)$ and $(+)$ alleles respectively at the polymorphic site $A$ in COLIA2 (EcoRI $\times N F 3)$. Control 1 is a known heterozygote, control 2 is a $(++)$ homozygote. recombination fraction between the markers and the mutation, presumably somewhere in the collagen gene, was given as 0.0005 . This figure, used for all collagen 1 marker systems, was a conservative estimate from an average value for the recombination fraction of $0.01 \mathrm{cM}$ per megabase adjusted for the $50 \mathrm{~kb}$ of DNA which includes the genes and flanking regions. There is no evidence of recombination 'hot spots' at either locus.

\section{PEDIGREE B}

This pedigree is illustrated because it is typical of those that are too small to derive an independent high probability of linkage to either locus. Therefore, the probability that the disease in this family is linked to collagen 1 genes at all must be used in the risk calculations. The large study referred to above ${ }^{11}$ showed no evidence of a third OI locus and estimated that the proportion of linked families lay between 0.91 and 1.00 using a confidence limit of $95 \%$. The most likely value for the proportion of linked families derived from the data is 0.98 . The figure used in the risk calculation was 0.95 , lower than the most likely value but higher than the lower limit of the $95 \%$ confidence interval.

The second problem in this family was to identify the mutant locus. The phenotype was fairly typical Sillence IA and the large study had found that of the 21 Sillence IA families where the concordant locus could be defined, 14 were linked to $C O L 1 A 1$ and seven to COL1A2. Though the chances were thus in favour of linkage to $C O L 1 A 1$, it was obviously wrong to assign the disease in this family on that basis alone. The approach we adopted was to try to exclude one of the loci by showing a recombinant. The probability that the other, concordant, locus was the mutant would then be equal to the prior probability that the family was linked to one or other of the collagen 1 genes and taken to be $\mathbf{0 . 9 5}$. The final risk can then incorporate the additional information provided by meioses at the concordant locus using Bayes's formula.

Examination of the pedigree shows that at $C O L 1 A 2$ allele 3 has been inherited by all three children in generation II although only the proband (II.2) and his brother (II.1) had inherited the OI phenotype. COL1A2 could only be excluded as the mutant locus if the apparently unaffected sib (II.4) had not inherited the OI gene. He was very carefully examined for signs of the disease and none was found. On this basis, it was deduced that COL1A1 was the mutant locus and, further, that allele 2 at that locus was segregating with the disease. The pedigree contained three fully informative, non-recombinant meioses at COL1A1 and, obviously, no recombinants. This conditional information was used to produce a final risk of 0.987 that the disease was linked to COL1AI (table 2). 
Table 2 Calculations of the probability that families $B$ and $D$ are linked to the non-discordant collagen 1 locus using Bayes's formula.

\begin{tabular}{llc}
\hline & Linked & Not linked \\
\hline & Family B $($ COLIAI) \\
Prior probability & 0.95 & 0.05 \\
Conditional probability & 1.00 & 0.25 \\
3 n-r, 0 r meioses & 0.95 & 0.0125 \\
Joint probability & 0.987 & 0.013 \\
Final probability & Family D $(C O L 1 A 2)$ \\
& 0.95 & 0.05 \\
Prior probability & 1 & $2.44 \times 10^{-4}$ \\
Conditional probability & & $1.22 \times 10^{-5}$ \\
Z (0.00)=3.60 & 0.95 & $1.28 \times 10^{-5}$ \\
Joint probability & 0.9999872 & \\
Final probability & & \\
\hline
\end{tabular}

The COL1Al genotypes of the proband and his wife made all meioses fully informative and the COLlAl genotype of the fetus showed that it had inherited the normal allele from the proband. Therefore, the risk of its having inherited the OI gene was reduced from $50 \%$ to $0.65 \%$, this residual risk being due to the chance that the disease in this family is not linked to either collagen 1 locus. Informed of this risk, the parents decided to continue with the pregnancy and a normal child was born at term.

\section{PEDIGREE C}

The structure of family $\mathrm{C}$ precluded precise identification of the mutant locus because it contained only a single meiosis. Evidently this event could not have provided sufficient information to exclude either locus. Even so, it might have been possible to identify a normal allele at each locus in the proband as long as the parental genotypes were completely informative, in which case the one in four of future pregnancies that inherited both these alleles would not have inherited the OI gene. The parents were so concerned to have an unaffected child that they were prepared to terminate three out of four of pregnancies knowing that, of these, only one out of three was sure to be affected, having inherited the same two alleles from the proband as their affected child (II.1). The remaining two out of three carried only a $50 \%$ risk of having inherited the same allele as the affected child at only one locus.

In the event, the proband was homozygous for all markers at $C O L I A I$ so even this exclusion prenatal diagnosis was not possible.

\section{PEDIGREE D}

This three generation family was completely genotyped at both COLIAI and COLIA2 and the mutant allele identified as $C O L 1 A 2$, allele 3 segregating with a lod of $3.60(\theta=0.00)$. Since this pedigree, unlike pedigree $A$, was not used in the large study which established the 0.95 risk figure for linkage, the concordant meioses could legitimately be used as conditional information to produce a final risk for linkage to COL1A2 of 0.9999872 (table 2). Very unfortunately, the only member of the pedigree to be homozygous for all markers at COL1A2 was the proband whose request for prenatal diagnosis had initiated the segregation analysis. However, other members of the family who are informative at the mutant locus have requested prenatal diagnosis for future pregnancies and will benefit from the complete analysis of the pedigree even though, until other informatives are found, the proband will not.

\section{Discussion}

These four pedigrees were chosen to illustrate some of the features associated with prenatal diagnosis of $\mathrm{OI}$ by identification of the mutant collagen 1 alleles. OI differs from all other diseases for which prenatal diagnosis is currently practised in that two loci are consistently involved rather than only one. Though some other disorders, notably thalassaemia and haemophilia, are caused by mutations at $\alpha$ and $\beta$ globin or factor VIII and factor IX loci, there is no problem in deciding which is the mutant locus from simple clinical observations or biochemical tests or both. This is not yet the case in OI and though there are correlations between some aspects of the phenotype and the mutant collagen 1 locus they are too insecure to be used on their own for prenatal diagnosis. ${ }^{11}$

The inability to decide in advance whether the disease is linked to COL1A1 or COL1A2 means that a family study must be undertaken to find one or more convincing recombinants at one of the loci and from this infer that the concordant locus is the mutant. Having identified the OI locus, the concordant allele must be identified in the usual way. The practical result of these preliminaries is that the family study has usually to be more extensive than is the case for other single locus diseases when it is only necessary to test sufficient meioses to identify the mutant allele, the mutant locus being known.

Pedigrees $\mathrm{A}$ and $\mathrm{D}$ are sufficiently large and informative that the mutant locus is beyond dispute. Even so, the couple requesting prenatal diagnosis cannot always be helped if, as in pedigree $\mathrm{D}$, the affected family member is homozygous for all markers at the mutant locus. They must wait for further markers that can distinguish his alleles. Though no use to him at present, other heterozygous family members are now in the position to be offered prenatal diagnosis thanks to the family study which his request initiated.

In smaller pedigrees, the certainty with which the mutant locus is identified diminishes as fewer meioses 
are available both to reveal recombinants at the unlinked collagen 1 locus and to build up a high lod score at its concordant partner. Under these circumstances, as in pedigree $B$, the risk calculation begins with the prior probability that the family is linked to either of the genes. This is derived from the estimate of the proportion of 'collagen 1 linked' OI families, as opposed to 'collagen 1 unlinked' families, in the population from which the family in question was drawn. An international multicentre study of 38 families estimated this proportion as being between 0.91 and 1.00 at $95 \%$ confidence with no direct evidence of any unlinked families and a maximum likelihood of $0 \cdot 98 .{ }^{11}$ Inevitably the populations from which these 38 families were drawn were very different, so the study recommends a figure of 0.95 for the prior probability that a new dominant OI family, of whatever Sillence type, is linked to one or other of the collagen 1 genes.

Since identification of one collagen 1 locus as the mutant depends on showing discordance at the other, the recombinants used to do this assume a special importance. It is therefore essential to be satisfied that the phenotype and paternity/maternity information used to identify a recombinant meiosis is correct, otherwise there is a danger, especially in small families, of a prenatal diagnosis being based on identifying alleles at the wrong locus.

Additional informative meioses at the concordant locus can then, and only then, be used as further evidence in favour of linkage. Once phase is established, each fully informative, non-recombinant meiosis reduces by one half the conditional probability that the disease is not linked to the concordant locus. This conditional probability is also equal to the inverse of the odds ratio in favour of linkage and can therefore be derived directly from the lod score where this is more convenient.

We thank our colleagues, Drs $O$ Jensson and $C$ Berry, for permission to report patients under their care. The work was supported by grants from Action Research for the Crippled Child and the Brittle Bone Society (UK). C Baigrie is a Nuffield Trust Scholar.

1 Byers PH, Bonadio JF, Cohn DH, et al. Osteogenesis imperfecta: the molecular basis of clinical heterogeneity. Ann NY Acad Sci 1988;543:117-28.

2 Sillence DO, Senn A, Danks DM. Genetic heterogeneity in osteogenesis imperfecta. F Med Genet 1979;16:101-16.

3 Young ID, Thompson EM, Hall CM, Pembrey ME. Osteogenesis imperfecta type IIA: evidence for dominant inheritance. f Med Genet 1987;24:386-9.

4 Thompson EM, Young ID, Hall CM, Pembrey ME. Recurrence risks and prognosis in severe sporadic osteogenesis imperfecta. F Med Genet 1987;24:390-405.

5 Sykes BC. The molecular genetics of collagen. Bioessays 1985;3: 112-7.

6 Elejalde BR, Elejalde MM. Prenatal diagnosis of perinatally lethal osteogenesis imperfecta. Am $\mathcal{F}$ Med Genet 1983;14:353-9.

7 Shapiro JE, Phillips JA III, Byers PH, et al. Prenatal diagnosis of lethal perinatal osteogenesis imperfecta. $\mathcal{f}$ Pediatr 1982;100: $117-33$.

8 Tsipouras P, Myers JC, Ramirez F, Prockop DJ. Restriction fragment length polymorphism associated with the pro- $\alpha 2$ (1) gene of human type I procollagen. F Clin Invest 1983;72:1262-7.

9 Grobler-Rabie AF, Wallis G, Brebner DK, Beighton P, Mathew CG. Detection of a high frequency Rsal polymorphism in the human pro- $\alpha 2$ (1) collagen gene which is linked to an autosomal dominant form of osteogenesis imperfecta. EMBO f 1985;4: 1745-8.

10 Sykes BC, Ogilvie DJ, Wordsworth BP, Anderson DJ, Jones N. Osteogenesis imperfecta is linked to both type I collagen structural genes. Lancet 1986;ii:69-72.

11 Sykes BC, Ogilvie DJ, Wordsworth BP, et al. Consistent linkage of dominantly-inherited osteogenesis imperfecta to the type I collagen loci: COL1A1 and COL1A2. Am $\mathcal{J}$ Hum Gene 1990;46:293-307.

12 Ogilvie DJ, Wordsworth BP, Zoll B, Schmitke J, Dalgleish R, Sykes BC. Segregation of all four fibrillar collagen genes in the Marfan syndrome. Am $\mathcal{F}$ Hum Genet 1987;41:1071-82.

13 Ogilvie DJ, Aitchison K, Sykes BC. An RFLP close to the human collagen gene COL1A1. Nucleic Acids Res 1987;15:4699. 